\title{
Purpose and Enactment in Job Design: An Empirical Examination of the Processes \\ Through Which Job Characteristics Have Their Effects
}

\author{
Kevin Daniels \\ University of East Anglia \\ Jane Glover \\ University of Birmingham \\ Rachel Nayani \\ University of East Anglia \\ Nadine Mellor \\ Uside \\ Fehmidah Munir \\ Loughborough University
}

Acknowledgements: For their comments on this paper, we would like to thank Jennifer Lunt, Olga Tregaskis, Constanze Eib, Mariella Miraglia, Roberta Fida, Karina Nielsen and participants in the University of East Anglia's Employment Systems and Institutions Work in Progress seminars.

Disclosure Statement: The training described in Study 2 now forms part of a training package delivered by University of East Anglia staff to NHS workers and others as funded continuing professional development. There are no other conflicts of interest.

This paper will published as: Daniels, K., Glover, J., Nayani, R., Mellor, N., Munir, F. Purpose and enactment in job design: An empirical examination of the processes through which job characteristics have their effects. Policy and Practice in Health and Safety. This is not the copy of record. 


\title{
Purpose and Enactment in Job Design: An Empirical Examination of the Processes Through Which Job Characteristics Have Their Effects
}

\begin{abstract}
Job characteristics are linked with health, safety, well-being and other performance outcomes. Job characteristics are usually assessed by their presence or absence, which gives no indication of the specific purposes for which workers might use some job characteristics. We focused on job control and social support as two job characteristics embedded in the well-known DemandControl-Support model (Karasek \& Theorell, 1990). In Study 1, using an experience sampling methodology $(N=67)$ and a cross-sectional survey methodology $(N=299)$, we found that relationships between the execution of job control or the elicitation of social support and a range of other variables depended on the purposes for which job control was executed or social support elicited. In Study $2(N=28)$, we found that it may be feasible to improve aspects of well-being and performance through training workers on how to use job control or social support for specific purposes.
\end{abstract}

Keywords - job design; job characteristics; job control; social support; self-regulation. 


\section{Introduction}

Job design is prominent in international systems and guidance to protect workers from psychosocial risks associated with a range of psychological and psychosomatic harms (Cousins MacKay, Clarke, Kelly, Kelly \& McCaig., 2004; Dollard, Skinner, Tuckey \& Bailey et al., 2007). Job design is concerned with the activities of workers, their duties, the tasks required to perform their work, and how those tasks and duties are structured and scheduled (Morgeson \& Humphrey, 2008; Parker \& Ohly, 2008). Job design is described by job characteristics such as job demands, job control, skill use, task variety, role clarity, use of skills, variety in tasks, social support and social contact at work (see e.g., Cousins et al, 2004; Hackman \& Oldham, 1976; Karasek \& Theorell, 1990; Warr, 2007).

In policy guidance and much research, job characteristics are typically assessed by measures that gauge the presence or absence of features of work and these features are assumed to be relatively stable (Grant \& Parker, 2009) and to exist independently of the person performing the job (Daniels, 2011). Although there is consistent evidence of links between the absence or presence of job characteristics and health, safety and performance outcomes (Humphrey, Nahrgang \& Morgeson, 2007; Nahrgang, Morgeson \& Hofmann, 2011; Nieuwenhuijsen, Bruinvels \& Frings-Dresen, 2010; Stansfeld \& Candy, 2006; Theorell, Hammarström, Aronsson et al, 2015), this measurement approach leads to a number of problems.

In this paper, we address one set of problems related to the dynamic processes through which workers use certain job characteristics to regulate their own experience of work, wellbeing, health and safety, in turn leading to impoverished knowledge of the employment practices that can support workers to use those job characteristics effectively and as intended. To address this set of problems, our aim in the present paper is to examine whether the use of job 
characteristics for different reasons is empirically distinct from the presence of the same job characteristics. We do not aim to provide a new methodology or construct new scales. Rather, our aim is examine the importance of complementing existing methods for assessing job design and tools to augment job redesign by taking into consideration how workers can or should be using the characteristics of their jobs and the goals that they pursue.

We use job control and social support as focal job characteristics, because they feature in a prominent model of job design and health (Karasek \& Theorell's Demand-Control-Support model, 1990), they have been linked to safety (Nahrgang et al., 2011), and there has been prior work that indicates job control and social support are used by workers for the specific purposes of problem-solving and expressing affect (Daniels \& Harris, 2005; Daniels, Boocock, Glover, Hartley \& Holland, 2009; Daniels, Beesley, Wimalasiri \& Cheyne, 2013a; Daniels, Wimalasiri, Cheyne \& Story, 2011). Job control reflects workers' authority to make decisions and encompasses control over working schedules and objectives and social support at work is characterized by help from supervisors and coworkers (Karasek and Theorell, 1990).

Prior work on enacting job control and social support for specific purposes has focused on a small range of outcomes, usually within a single domain such as well-being or innovation, has used a single methodology (experience sampling) and has tended to focus on a single purpose (e.g. problem-solving). The present study is unique in that we use several methods, a wider range of purposes and a wider range of well-being and other outcomes encompassing different domains. This enables us to examine whether enacting a job characteristic for one purpose is empirically distinct from and has different outcomes to enacting the same job characteristic for another purpose. Moreover, we provide an explicit test of whether enacting a 
job characteristic for a specific purpose is empirically distinct from the availability of that job characteristic.

\section{Enacted Job Characteristics}

Most research and policy guidance on job characteristics requires workers to rate the presence or absence of specific job characteristics, either on Likert-type scales or frequency based scales (e.g. HSE Management Standards Indicator Tool, Cousins et al., 2004; Job Diagnostic Survey, Hackman \& Oldham, 1975; Job Content Questionnaire, Karasek, Brisson, Kawakami, Houtman, Bongers \& Amick, 1998; Copenhagen Psychosocial Questionnaire, Kristensen, Hannerz, Høgh \& Borg, V. 2005; Work Design Questionnaire, Morgeson \& Humphrey, 2006). There is a considerable volume of research showing associations between job characteristics assessed in this way and health, safety, wellbeing and performance outcomes (Humphrey et al., 2007; Nahrgang et al., 2011; Nieuwenhuijsen et al., 2010; Stansfeld \& Candy, 2006; Theorell et al., 2015). In spite of the success of this assessment approach for research, policy and practice, it has several problems that may make it sub-optimal and/or requiring supplementary information (Daniels, 2006, 2011).

One problem is that this approach to measurement ignores how workers may interpret the presence or absence of certain job characteristics in terms of their own personal goals and motivations. This problem has been addressed in research on how workers' beliefs about job characteristics moderate the impact of job characteristics on well-being (Daniels, Hartley \& Travers, 2007) and research that shows how met needs can mediate the relationship between job characteristics and well-being (Van den Broeck, Vansteenkiste, De Witte, \& Lens, 2008). A second problem is that this measurement approach ignores the day-to-day dynamic processes that underpin work contexts and the behavior of workers (cf. Peterson, 1998). This problem has been 
Purpose and enactment in job design 6

addressed through research on daily experiences in the workplace using experience sampling, diary and day reconstruction methods (see e.g. edited works by Xanthopoulou, Bakker \& Illies, 2012; Bakker \& Daniels, 2012).

A third problem, addressed in the present paper, is that the approach ignores the agency of workers in the processes that underpin the relationship between job design and various outcomes (Daniels, 2006, 2011). Worker agency has become more salient as an issue in order to understand how complex organizational systems function in the context of complex and unpredictable operating environments (Griffin, Neal \& Parker, 2007). In relation to health, safety and well-being, prominent job design models proposed that certain aspects of job design act as resources that enable workers to manage their own health, safety and well-being or attain other personally important goals (Demand-Control-Support model, Karasek \& Theorell's, 1990; Job Demands Resources Model, e.g., Bakker \& Demerouti, 2007). Thus, certain aspects of job design can allow workers the agency to self-manage health, safety and well-being. However, these models do not specify ways in which aspects of job design come to be resources used to self-manage health, safety and well-being.

The issue of how resources come to be used has been addressed in a general way by Feldman and Worline (2011), who propose that a resource becomes useful when it is bought into use. The process of bringing a resource into use therefore entails understanding behavior rather than understanding what resources are available. A focus on behavior is reinforced by Feldman and Worline's suggestion that the availability of a resource does not inevitably mean that the resource will be used. An available resource is labelled a potential resource by Feldman and Worline. Moreover, they argue that the purpose that a resource is used to fulfill determines what kind of resource the resource will become and that a single resource can be used and adapted for 
multiple purposes depending on context (for example, social support can be used for emotional venting or to gather advice on how to solve a problem).

To address the problem of agency in relation to job design, Daniels (2006) introduced the notion of enacted job characteristics, or job characteristics as they become manifest through the behavior of workers. For example, a job may be designed to allow workers autonomy over their schedules, but that autonomy becomes enacted when a worker decides to alter his/her work schedules. Reflecting the distinction between a behavior and the function or purpose of that behavior (Lazarus, 1999; Skinner, Edge, Altman, \& Sherwood, 2003, see also Feldman \& Worline, 2011), job characteristics may be enacted for specific purposes. For example a worker may exercise autonomy over work schedules to spend more time on solving a problem or to take a break from tasks that are particularly demanding.

Enacted job characteristics have something in common with the concept of job crafting (Daniels, 2012), in that both relate to the behavior of workers in job design. In this way, research that indicates the potential to train workers to craft better jobs may mean it is possible to train workers to enact aspects of their work for specific and adaptive purposes (Demerouti, Xanthopoulou, Petrou \& Karagkounis, 2017; van den Heuvel, Demerouti \& Peeters, 2015; Van Wingerden, Derks, \& Bakker, 2015). However, the two concepts differ: Job crafting refers to workers making future oriented changes to their job characteristics (Wrzesniewski \& Dutton, 2001), which may or may not then become enacted. Enacted job characteristics refer to existing job characteristics, and thus operate within existing work processes and systems. However, it may be possible to enact a job characteristic for proactive purposes (e.g., solve a problem in such a way that alters work processes to prevent the problem happening again) or for more reactive 
purposes in response to externally imposed events or demands or some mixture of the two (Griffin et al., 2007; Strauss, Griffin, Parker \& Mason, 2015).

Enacting job characteristics for different purposes leads to a fourth problem inherent in the standard approach to assessing job characteristics. The standard approach gives information on what job characteristics to change through job redesign. However, it gives very little indication of how job design can be integrated with other employment practices that also have a bearing on workers behaviors and their goals, such as training and performance management systems (Christina, Dainty, Daniels, Tregaskis, \& Waterson, in press). As suggested in sociotechnical design principles, job redesign should be integrated and aligned with other organisational processes and workers' goals (Cherns, 1987; Clegg, 2000). Moreover, the high performance work systems literature (Appelbaum, Bailey, Berg \& Kalleberg., 2000; Combs, Liu, Hall \& Ketchen, 2006) suggests that any job redesign that allows workers' to use their abilities, potentially including job redesign to allow use of abilities to self-manage health, safety and wellbeing, should be supplemented with employment practices to enhance workers' abilities (e.g., through training workers in coping skills) and motivation to use those abilities (e.g., through developing appropriate safety climate norms).

If there is no integration of job redesign with other employment practices, job redesign may have no effects on intended outcomes or may even have adverse effects (Daniels, Gedikli, Watson, Semkina \& Vaughn, 2017). For example, job control is held to contribute to workers' health by facilitating workers' problem-solving (Karasek \& Theorell, 1990), but if workers have not been trained to solve problems, a job redesign intervention to improve problem-solving through improving job control may not be successful. Therefore, it is important to link the enactment of a job characteristic explicitly to the purpose for which it is enacted. 


\section{Study 1 Introduction}

\section{INSERT FIGURE 1 HERE}

In Study 1, we test the idea that different job characteristics enacted for different purposes are distinct constructs with distinct correlations with other outcome variables. Figure 1 summarizes the hypotheses for Study 1. We examine the enactment of job control and social support. We examine the following purposes for enacting job control or social support: problemsolving, taking breaks from work and switching from primary or workers' main or priority tasks to secondary tasks, subsidiary or lower priority tasks. We examine these purposes because in the Demands Control Support model (Karasek \& Theorell, 1990), problem-solving and taking breaks from work are held to be important ways in which workers can regulate their own wellbeing and health at work, and switching from primary to secondary work tasks is an alternative to taking a complete break from work if a break is needed from a primary task (Elsbach \& Hargadon, 2006; Fritz, Lam \& Spreitzer, 2011). Therefore, in Study 1, we propose have a sixfold typology reflecting different combinations of the execution of job control or elicitation of support for three different purposes, so that the following comprise separate constructs (Hypothesis 1a): i) job control used for problem-solving (CON-PROB); ii) job control used to take a complete break from work (CON-BREAK); iii) job control used to switch from primary work activities to secondary work activities (CON-SWITCH TASKS); iv) social support used for problem-solving (SUP-PROB); v) social support used to take a complete break from work (SUPBREAK); vi) social support used to switch from primary work activities to secondary work activities (SUP-SWITCH TASKS). Moreover, we expect the presence or absence of job control and social support to be distinct from the enactment of job control and social support for these three specific purposes (Hypothesis 1b). 
Job control or social support cannot be enacted if they are not present, so we expect that compared to the presence of social support, the presence of job control is more closely related to job control enacted for specific purposes (Hypothesis 2a). Also, we expect that compared to the presence of job control, the presence of social support is more closely related to social support enacted for specific purposes (Hypothesis 2b). There is evidence that job control may allow workers to spend discretionary time with co-workers (Oldham \& Rotchford, 1983) and to seek support for problem-solving (Daniels, Glover, Beesley, Wimalasiri, Cohen \& Cheyne, 2013b). Therefore we expect that executing job control for a specific purpose may enable workers to seek support to achieve that same purpose (Hypotheses $3 \mathrm{a}, 3 \mathrm{~b}, 3 \mathrm{c}$ ).

Our final set of hypotheses are based on CON-PROB, CON-BREAK, CON-SWITCH TASKS, SUP-PROB, SUP-BREAK and SUP-SWITCH TASKS having distinctive relationships with other variables. First, CON-PROB and SUP-PROB are concerned with problem-solving, and so we would expect a relationship with levels of experienced problem-solving demands (Hypothesis 4a). We also expect CON-PROB and SUP-PROB to be more closely related with creativity (the generation of new and useful ideas, George, 2007) (Hypothesis 4b) given the relationship between problem-solving in general and creativity (Scott \& Bruce, 1994). Creativity through problem-solving is also facilitated by incubation. Incubation occurs when there is a temporary shift in cognitive processes away from a task (Sio \& Ormerod, 2009). Sio and Ormerod suggest that incubation is a largely unconscious process that: enables extensive search through memory for information relevant to the task; allows insightful recombination of existing knowledge into new knowledge structures; enables selective forgetting of information less relevant to the issue; and allows individuals to restructure their thoughts about the task. Because incubation is an unconscious process, it is more likely to occur when individuals shift their 
attention away from the task. One way to divert conscious processes away from a task is to engage in secondary work activities (Elsbach \& Haragdon, 2006). Therefore, we expect CONSWITCH TASKS and TAL-SW to be correlated with creativity (Hypothesis 4b).

In the Demands Control Support model, learning is thought to mediate the links between job control or social support used for problem-solving (Karasek \& Theorell, 1990), and there is evidence that on-the-job learning does mediate between well-being and enacting job control or social support for problem-solving (Daniels et al., 2009). Therefore, we do not expect a close relationship between indicators of well-being and either CON-PROB or SUP-PROB. However, CON-BREAK and SUP-BREAK may maintain workers' well-being and reduce fatigue because breaks allow workers to detach themselves from work, recover their energy, or repair their affective experience (Miner, Glomb, \& Hulin, 2005; Sonnentag \& Fritz, 2015) (Hypothesis 4c). In the present study, we focus on negative and positive affect as core affective indicators of wellbeing (Watson \& Tellegen, 1985). Switching to secondary work tasks involves continuing effort and does not allow respite from work, and therefore may have a detrimental impact on wellbeing and fatigue (Hypothesis $4 \mathrm{~d})$.

Hypotheses 4a to 4d make predictions for enacting job control and social support for specific purposes. However, if the enactment of job control or social support for a specific purpose is different from the availability of job control or social support, then we would expect there to be differences in the relationships between the availability of job control or social support with a range of other variables compared to the enactment of job control or social support for specific purposes (Hypotheses 4e, 4f).

Although Hypotheses $4 \mathrm{a}$ to $4 \mathrm{~d}$ make distinct predictions about different reasons to enact a job characteristic, the Hypotheses make the same predictions for job control used to fulfill a 
given purpose as social support used to fulfill the same purpose. On the one hand, if similar patterns emerge for enacted job control as enacted social support, there is evidence for equifinality, and that job control and social support may substitute for one another in job design. On the other hand, if there is a differential pattern of relationships, it would mean job control and social support are not substitutable but may be complementary. Indeed, the reasoning underpinning Hypotheses $3 \mathrm{a}$ to $3 \mathrm{c}$ would suggest enacting job control allows for the enactment of social support. Therefore, we examine whether relationships with other variables are different for job control enacted for specific purposes and social support enacted for specific purposes (research question 5).

\section{Study 1 Methods}

We used both an experience sampling methodology (ESM) and a cross-sectional survey. ESM allows capture of data to examine short-term fluctuations in phenomena and within-person differences. A cross-sectional survey allows examination of data over longer term frames, between-person differences and places much less burden on participants. In both samples, scale scores were calculated by summing the scores for each item and dividing by the number of items in the scale.

\section{ESM Sample and Procedure}

Participants $(N=71)$ were volunteers from three different organizations (an automotive company $N=25$, a management consultancy $N=25$, a large retail firm $N=21$ ). All participants were knowledge workers (e.g., design engineers, analysts, planners). Most of the sample (55\%) was male. The average age was 31.8 years $(S D=8.5)$. Personal digital assistants (PDAs) administered questionnaires four times daily (10.30 a.m., 12.30 p.m., 2.30 p.m., and 4.30 p.m.) over one working week (Monday to Friday). The PDAs' alarm indicated when the questionnaire 
was to be completed. Participants provided complete data on 693 occasions. After taking into account known absences (scheduled leave, etc.), the average compliance rate was $58 \%$ (SD = $23 \%$, range $7 \%$ to $100 \%$ ). Although the overall compliance is low, prior to the study, participants from the management consultancy (average compliance $=44 \%$ ) indicated they spent much time on the telephone to clients, so they anticipated a low compliance rate as calls with clients could not be interrupted. Compliance rate was unrelated to any of the substantive variables $(p>.10)$. We considered the sample size and number of observations adequate, given the availability of algorithms for conducting confirmatory factor analyses with ESM data (Hypothesis 1, see below), because the number of observations provides good statistical power (Hypotheses 2-4, Research Question 5) and because of a larger sample size for the survey phase of Study 1. Because of repeated sampling, ESM can place a considerable burden on participants. Therefore, to reduce this burden, we did not ask questions about experiences of the availability of job control or social support at each measurement point. Instead and prior to the ESM phase, participants completed a questionnaire about their usual availability of job control and social support. We did not consider this a problem, because the traditional approach to assessing job characteristics assumes the presence of job control and social support to be relatively stable.

For variables assessed with ESM, between $51 \%$ and $87 \%$ of the variance could be attributable to variation between observations, between $13 \%$ and $47 \%$ between individuals and between $0 \%$ and $13 \%$ between organizations. For variables assessed by questionnaire, some $96 \%$ was between individuals for availability of job control and $87 \%$ for availability of social support, with the remainder being attributable to differences between organizations.

Availability of job control and social support. Job control $(\alpha=.82)$ was assessed with six items adapted from Breaugh (1985) (e.g., "Can you control the sequencing of your work 
activities?"). Two items in this scale each tapped three facets of job control; control over work schedules, work processes, and work objectives. Social support $(\alpha=.80)$ was assessed with four items adapted from items used by Daniels (2000) (e.g., "Can you confide in other people at work?"). Items were rated on a 5-point scale $(1=$ never, 5 = very often $)$.

\section{ESM Measures}

Problem-solving demands. We assessed problem-solving demands with the question "In the past hour, how many issues without an obvious answer or solution have you had to deal with?". Demands were rated on a 6-point scale (0, 1, 2, 3, 4, 5 or more). Daniels et al. (2009) provide evidence for the validity of this single item scale.

Fatigue and affect. We asked participants how they felt at that moment in time with items validated for organizational contexts (Daniels, 2000). Items were rated in a five-point scale $(1=$ not at all, $5=$ very). Fatigue $(\alpha=.90)$ was assessed with the items "fatigued" and "tired"; NA $(\alpha=.85)$ by "anxious" and "worried"; and PA $(\alpha=.88)$ by "motivated" and "enthusiastic".

Creativity. We assessed creativity with two items (Daniels et al., 2011, $\alpha=.87$; "In the past hour, have you had ideas that could help you deal with difficult issues more efficiently?", "In the past hour, have you had ideas that could help you solve work problems more quickly?"). Items were rated on a 5-point scale $(1=$ no, $2=y e s$, one idea, $3=y e s$, two ideas, $4=$ yes, three ideas, 5= yes, four or more ideas).

Enacted job control and social support. Participants rated the previous hour's activities on 6-point fully anchored scales $(1=$ not at all, $6=$ to a large extent $)$. Each scale consisted of two items and scores calculated by summing item scores and dividing by two. CON-PROB and SUP-PROB were assessed with items developed by Daniels and colleagues (Beesley, Cheyne, \& Wimalasiri, 2008; Daniels et al., 2009). If a participant reported no problem-solving demands in 
a given hour, data were excluded from the analyses for that hour. CON-PROB and SUP-PROB had good reliability ( $\alpha=.84$ and $\alpha=.88)$. Example items are "In the past hour, did you change your work objectives for the hour to solve the issues?" for CON-PROB and "In the past hour, did you discuss the issues to help you solve them?" for "discussing problems with others to solve problems" for SUP-PROB.

CON-BREAK $(\alpha=.76)$ was assessed with the items "In the past hour, did you change the order in which you normally do your work tasks to take a complete break from work?" and "In the past hour, did you change your work objectives for the hour to enable you to take a complete break from work?". SUP-BREAK $(\alpha=.81)$ was assessed with the items "In the past hour, did you talk to other people to give you a complete break from work?" and "In the past hour, did you discuss things not related to work with other people?". CON-SWITCH TASKS $(\alpha=.80)$ was assessed with the items "In the past hour, did you change the order of your work tasks to do work activities not directly related to your main work task for the hour?" and "In the past hour, did you change your work objectives for the hour to do work activities not directly related to your main work task for the hour?". SUP-SWITCH TASKS $(\alpha=.78)$ was assessed with the items "In the past hour, did you talk to other people about work activities not directly related to your main work task for the hour?" and "In the past hour, did you discuss things about work not related to your main work task for the hour?".

Following the precedent of previous studies on the enactment of job control and social aspects of the work environment for specific self-regulatory purposes such as problem-solving (Daniels et al., 2009, 2011, 2013b; Daniels, Glover \& Mellor, 2014), the items used in these six scales link enactment of job control or talking to co-workers (e.g., changing the order of tasks and schedules) with the purpose of enacting control or support (break or switch to work activities 
not related to main activity for the hour). It is important to operationalize such links to understand precisely how job control and social support have their effects depending on the purpose for which they are enacted (see Daniels et al., 2009, 2011, 2013b, 2014).

\section{Cross-Sectional Survey Sample and Procedure.}

Participants $(N=299)$ were knowledge workers (e.g., scientific project officer, software engineer) from two organizations (a large public sector organization, $N=128$, response rate $6 \%$; and a smaller private sector information systems company, $N=171$, response rate $38 \%$ ). Questionnaires were administered via a web-link distributed by email. In the public sector organization, emails were distributed to labor union members by a senior union official. In the private sector organization, emails were distributed by a senior manager. The response rate from the public sector organization was low. Surveys distributed via a web-link can suffer from low response rates and the distribution via a union official may have less impact than distribution via a senior or line manager. Notwithstanding, convergence of results with the ESM data is the strongest indication that low response rates has not biased the results (Rogelberg \& Stanton, 2007). Some $71 \%$ of the sample were male; $32 \%$ of the sample were 35 years of age or younger; $29 \%$ were aged $36-45$; and the remainder aged 46 or over.

\section{Cross-Sectional Survey Measures}

For each variable, scale scores were calculated by summing the scores for each item and dividing by the number of items in the scale.

Availability of job control and social support. Job control $(\alpha=.82)$ was assessed with six items adapted from Breaugh (1985) (e.g., "In the past week, could you control the sequencing of your work activities?"). Two items in this scale each tapped three facets of job control; control over work schedules, work processes, and work objectives. Social support $(\alpha=.80)$ was assessed 
with ten items adapted from items used by Daniels (2000) (e.g., "In the past week, could you confide in other people at work?"). Items were rated on a 5 -point scale $(1=$ never, $6=$ very often).

Fatigue and affect. Participants were asked how they felt at work during the previous week (Daniels, 2000). Items were rated on a six-point fully anchored scale $(1=$ never, $6=$ all of the time). Three items each assessed fatigue ( $\alpha=.86$, e.g., "tired"), NA ( $\alpha=.89$, e.g., "worried") and PA ( $\alpha=.88$, e.g., "enthusiastic").

Creativity. We assessed creativity with three items (Daniels et al, 2011, $\alpha=.90$; e.g., "In the past week, how often did you have ideas that could improve your work performance?"). Items were rated on a 5 -point scale $(1=$ never, $5=$ very often $)$.

Enacted job control and social support. Two item scales were adapted from those used in the ESM phase to be relevant to the previous week (e.g., "In the past week, did you change the order in which you normally did your work tasks to allow you to take an unscheduled break during the working day?"). Ratings were made on a 5-point scale $(1=$ never, $5=$ very often $)$. Reliability for the scales was acceptable: CON-PROB $(\alpha=.77)$; SUP-PROB $(\alpha=.78)$; CONBREAK $(\alpha=.82)$; SUP-BREAK $(\alpha=.75)$; CON-SWITCH TASKS $(\alpha=.82)$; SUP-SWITCH TASKS $(\alpha=.86)$.

\section{Analysis}

Hypotheses 1a and $1 \mathrm{~b}$ were examined using CFA. Because of the nested nature of the ESM data (observations nested in people), Hypotheses 1a and 1b were examined using multilevel confirmatory factor analysis (ML-CFA) to separate within- from between-person variance. Maximum likelihood estimators can encounter problems with small numbers at the between-person level, especially where models are complex. These problems can be addressed 
with Weighted Least Squares Estimators (Hox, Maas \& Brinkhuis, 2010) and Bayesian estimation (Muthén, 2010). For Weighted Least Squares estimation, we used the WLSMV estimator. The WLSMV does provides conventional CFA fit statistics, and we used the Confirmatory Fit Index (CFI) and Root Mean Square Error of Approximation to assess model fit (RMSEA), as recommended by Williams, Vandenberg and Edwards (2009). Bayesian CFA does not use conventional CFA fit statistics. Parsimony or fit was assessed for each model by using three indices (Muthén, 2010): Potential Scale Reduction (PSR), where a level less than 1.1 indicates convergence, but not necessarily fit; Posterior Predictive Checking (PPC), which should ideally be non-significant $(p>.05)$, to indicate fit; Deviance Information Criterion (DIC) indicates a better fit, the smaller it is compared to other models. For the cross-sectional survey data, we used robust Maximum Likelihood Estimation to counter any problems with nonnormality in the data (Byrne, 2012). We used the CFI and RMSEA to assess model fit.

Hypotheses $1 \mathrm{a}$ and $1 \mathrm{~b}$ imply that items assessing the availability of job control, the availability of support, CON-PROB, SUP-PROB, CON-BREAK, SUP-BREAK, CON-SWITCH TASKS and SUP-SWITCH TASKS will form separate factors. We compared the fit of this hypothesized model with two alternative models. The first was a model that had separate factors for the availability of job control, the availability of support but had single factors for each function, namely problem-solving (consisting of all CON-PROB and SUP-PROB items), taking a complete break (consisting of all CON-BREAK and SUP-BREAK items) and switching activities (consisting of all CON-SWITCH TASKS and SUP-SWITCH TASKS items). The second model loaded the availability of job control, CON-PROB, CON-BREAK and CONSWITCH TASKS items on a single factor and the availability of social support, SUP-PROB, SUP-BREAK and SUP-SWITCH TASKS on a single factor. 
Hypotheses 2 through 4 were examined with correlations. For the ESM data, we analyzed within-person and between-person variance separately. For within-person variance, we centered each person's data at that person's mean and examined within-person correlations using the person mean centered data. For between-person variance, we examined correlations between the each person's average on each variable. For the ESM data, for analyses involving CON-PROB and SUP-PROB, data were analyzed only if participants reported problem-solving demands in a given hour.

\section{Study 1 Results}

Both the ESM and survey data supported the hypothesized Model. For the WLSMV estimation with the ESM data, the hypothesized model had best fit $(\mathrm{CFI}=.94$, RMSEA $=.02$, one alternative model $\mathrm{CFI}=.86$ and $\mathrm{RMSEA}=.04$, the other alternative model did not converge). All factor loadings were significant and in the hypothesized direction $(p<.05)$. For the ESM data, all Bayesian models showed good convergence (PSR =1.02). Although PPC was significant $(p<.01)$ for all models, the DIC was lowest for the hypothesized model $(26245.26$, alternative models, DIC > 26968). All factor loadings were significant and in the hypothesized direction at within- $(p<.01)$ and between-person levels of analysis (all $p<.01$, except one of Breaugh's job control items, $p<.06$ ). For the survey data, the hypothesized model had best fit $(\mathrm{CFI}=.92, \mathrm{RMSEA}=.06$, alternative models $\mathrm{CFI}<.79$ and RMSEA $>.09)$. All factor loadings were significant and in the hypothesized direction $(p<.01)$. Therefore, Hypotheses $1 \mathrm{a}$ and $1 \mathrm{~b}$ are supported as the results indicate that job control or social support enacted for different purposes reflect separate constructs, which are in turn different constructs from the availability of job control or social support. 
Tables 1 and 2 show the results of the descriptive statistics and correlations for both data sets.

\section{INSERT TABLES 1 AND 2 HERE}

$\mathrm{H} 2 \mathrm{a}$ states that the availability of job control should be more highly correlated with job control used for specific purposes than social support used for specific purposes. H2b states that the availability of social support should be more highly correlated with social support used for specific purposes than job control used for specific purposes. We were able to use betweenperson aggregated data for the ESM sample and the survey sample to examine $\mathrm{H} 2 \mathrm{a}$ and $\mathrm{H} 2 \mathrm{~b}$. For the ESM sample, Table 1 shows that there is only one significant correlation between the availability of job control and social support and the enactment of either for specific purposes and that correlation is not consistent with $\mathrm{H} 2 \mathrm{a}$ or $\mathrm{H} 2 \mathrm{~b}$ (job control and SUP-SWITCH TASKS, $r$ $=.28, p<.05)$. However, the availability of social support is negatively, if not significantly, correlated with CON-PROB, CON-BREAK and CON-SWITCH TASKS. Table 2 shows that job control is significantly correlated with CON-PROB, CON-BREAK and CON-SWITCH TASKS and these correlations are larger than the corresponding correlations with the availability of social support. The converse is the case for correlations involving availability of social support and SUP-PROB, SUP-BREAK and SUP-SWITCH TASKS.

Using Lee and Preacher's calculator for comparing correlations from the same sample (2013), we compared the correlations between the availability of job control and social support with CON-PROB, CON-BREAK and CON-SWITCH TASKS for H2a, and SUP-PROB, SUPBREAK and SUP-SWITCH TASKS for H2b. For H2a, we found that the availability of job control was more positively related to the enactment of job control for specific purposes than the availability of social support in two of out three cases with the ESM data and one out of three 
cases with the survey data $(p<.05)$. For $\mathrm{H} 2 \mathrm{~b}$, we found that the availability of social support was not more positively related to the enactment of social support for specific purposes than the availability of job control in the ESM data, but was more positively related in two out of three cases in the survey data $(p<.05)$. In summary, although the results are equivocal, the pattern of correlations and differences between correlations does tend towards support for $\mathrm{H} 2 \mathrm{a}$ and $\mathrm{H} 2 \mathrm{~b}$.

$\mathrm{H} 3 \mathrm{a}, \mathrm{H} 3 \mathrm{~b}$ and $\mathrm{H} 3 \mathrm{c}$ indicated that job control enacted for a specific purposes would be more closely related to social support enacted for the same purpose than social support enacted for different purposes. Tables 1 and 2 show that CON-PROB has higher correlations with SUPPROB than with SUP-BREAK or SUP-SWITCH TASKS for the within-participants' data of the ESM, between participants' data of the ESM and the survey data. The correlations between CON-PROB and SUP-PROB were significantly higher is three out of six cases $(p<.01)$. CONBREAK has higher correlations with SUP-BREAK than with SUP-PROB or SUP-SWITCH TASKS for the within-participants' data of the ESM, between participants' data of the ESM and the survey data. The correlations between CON-BREAK and SUP-BREAK were significantly higher is five out of six cases $(p<.01)$. CON-SWITCH TASKS has higher correlations with SUP-SWITCH TASKS than with SUP-PROB or SUP-BREAK for the within-participants' data of the ESM, between participants' data of the ESM and the survey data. The correlations between CON-SWITCH TASKS and SUP-SWITCH TASKS were significantly higher in all six cases $(p<.01)$. In summary, the results tend to support H3a and H3b, and offer unequivocal support for $\mathrm{H} 3 \mathrm{c}$.

$\mathrm{H} 4 \mathrm{a}, \mathrm{H} 4 \mathrm{~b}, \mathrm{H} 4 \mathrm{c}$ and $\mathrm{H} 4 \mathrm{~d}$ proposed specific relationships between job control and social support enacted for specific purposes and a range of other variables. H4a was generally supported, in that CON-PROB was associated with problem-solving demands for two out of 
three of the data types used, and SUP-PROB was associated with problem-solving demands in for all data types. $\mathrm{H} 4 \mathrm{~b}$ received some support, in that creativity was associated with CON-PROB, SUP-PROB, CON-SWITCH TASKS and SUP-SWITCH TASKS for the within-participants' data from ESM, and CON-PROB and CON-SWITCH TASKS was associated with creativity in the survey. H4c received less support. For the within-participants data from the ESM, only SUPBREAK was associated with less fatigue, less NA and more PA. Neither CON-BREAK nor SUP-BREAK were associated with fatigue or NA for the other data types. SUP-BREAK had no relationship with PA in the between-participants' data from the ESM and was negatively associated with PA in the survey. H4d received some support: CON-SWITCH TASKS and SUPSWITCH TASKS were both associated with NA for the within-participants data from the ESM and fatigue in the survey data. CON-SWITCH TASKS was associated with NA in the survey data.

H4e proposed the availability of job control has different sized correlations with a range of other variables than CON-SP, CON-BREAK and CON-SWITCH TASKS. H4f proposed the availability of social support has different sized correlations with a range of other variables than SUP-SP, SUP-BREAK and SUP-SWITCH TASKS. Using correlations with problem-solving demands, creativity, fatigue, PA and NA, we made paired comparisons using the betweenparticipants' data from the ESM and the survey data. For job control $(\mathrm{H} 4 \mathrm{e})$, we found differences in the size of the correlations between the availability of job control and CON-PROB, CONBREAK or CON-SWITCH TASKS in four out of 15 cases in the ESM data and 12 out of 15 cases with the survey data $(p<.05)$. For social support $(\mathrm{H} 4 \mathrm{e})$, we found differences in the size of the correlations between the availability of social support and SUP-PROB, SUP-BREAK or SUP-SWITCH TASKS in none out of 15 cases in the ESM data and 10 out of 15 cases with the 
survey data $(p<.05)$. The ESM data tend to support H4e but not H4f. The survey data support $\mathrm{H} 4 \mathrm{e}$ and $\mathrm{H} 4 \mathrm{f}$.

Finally RQ5 asked whether job control enacted for a specific purpose had a different relationships with other variables than social support enacted for that same purpose. We made pairwise comparisons for CON-PROB and SUP-PROB, CON-BREAK and SUP-BREAK and CON-SWITCH TASKS and SUP-SWITCH TASKS on the size of their correlations with problem-solving demands, creativity, fatigue, NA and PA. For CON-PROB and SUP-PROB, the size of the correlations differed significantly in three out of five cases with within-participants data from the ESM, one case for between-participants data and two cases for the survey data. For CON-BREAK and SUP-BREAK, the size of the correlations differed significantly in two out of five cases with within-participants data from the ESM, two cases for between-participants data and three cases for the survey data. For CON-SWITCH TASKS and SUP-SWITCH TASKS, the size of the correlations differed significantly in one out of five cases with within-participants data from the ESM, four cases for between-participants data and three cases for the survey data. Some 21 comparisons revealed significantly different correlations out of a total of 45 comparisons. Therefore, the results indicate that job control enacted for a specific purpose does have different relationships with other variables than social support enacted for that same purpose, further reinforcing the need to differentiate which job characteristics are enacted for which purposes.

\section{Study 1 Discussion}

In general, the results from Study 1 show that executing job control for a specific purpose forms a construct that is both distinct from the availability of job control and distinct from enacting job control for another purpose. The results also show that eliciting social support for a specific purpose is different from the availability of social support. These results were evident 
from CFAs and the pattern of correlations in two samples. Results tended to be less supportive of the Hypotheses when the between-participants' data were used from the ESM. This can be attributed to lower statistical power resulting from a small sample size $(N=71)$ relative to the larger number of observations in the within-participants' data (up to 693) or the survey data $(N=$ 299). One other unusual set of results concerns SUP-BREAK and fatigue and well-being. Although SUP-BREAK was associated with less fatigue, less NA and more PA in the withinparticipants' data in the ESM, SUP-BREAK was associated with less PA in the survey. This could suggest a reversing of relationships if variables are assessed over different time periods. In this case, taking a within-day work break with others might be beneficial for fatigue and wellbeing over a brief time frame, but if it becomes a behavior enacted over several days, it may lead to disengagement from work.

The pattern of correlations with variables such as problem-solving demands, creativity, fatigue and well-being indicates that the factoral distinctiveness of the availability of a job characteristic and its enactment for a specific purpose is not a trivial distinction: Different substantive outcomes could be obtained to those anticipated if job control or social support were enhanced in a workplace without attending to how workers would be expected to use job control or social support. A number of employment practices that influence the motivation or ability of workers to use job control or social support have potential to augment the effects of job control and social support (Appelbaum et al., 2000). However, given that workers are likely to be motivated to protect or enhance their own safety, health and well-being, interventions may have more potential if focused on enhancing the abilities of workers to use job control and social support more effectively. 
In this respect, training workers to use job control and/or social support for specific purposes could lead to intended outcomes. In Study 2, we tested this idea by evaluating an intervention directed at training workers to use job control and social support for more effective problem-solving. Study 2 therefore provides a test of the idea that workers enact job characteristics for specific reasons because, in Study 2, we attempted to effect changes in both the enactment of job characteristics and the reasons for enacting those job characteristics, but we did not attempt to effect changes in the availability of job characteristics. Moreover, because training is an employment practice and that directly seeks to change behaviors and/or cognitions, Study 2 provides an illustration of an employment practice that is linked directly to the enactment of job characteristics for specific purposes that can be used to supplement job redesign interventions to improve the availability of job characteristics. As such, the training was directed at the interface between the individual and his/her work, which is an approach that has been associated with higher success for interventions directed at the individual level (Stemmer, 2004).

The training focused on enhancing CON-PROB, SUP-PROB, CON-BREAK, SUPBREAK, CON-SWITCH TASKS and SUP-SWITCH TASKS in order to improve problemsolving directly (CON-PROB and SUP-PROB), problem-solving through encouraging incubation (CON-SWITCH TASKS, SUP-SWITCH TASKS) and respite from challenging work problems (CON-BREAK, SUP-BREAK). Given the focus of the training, as well as improvements in CON-PROB, SUP-PROB, CON-BREAK, SUP-BREAK, CON-SWITCH TASKS and SUP-SWITCH TASKS, we anticipated improvements in markers of problemsolving success (e.g., creativity), fatigue and well-being. Because markers of problem-solving success, especially learning (Karasek \& Theorell, 1990), should be related to well-being, we expected problem-solving success to mediate the relationship between the training intervention 
on the one hand and well-being and fatigue on the other. Moreover, because we trained workers to enact job control and social support, we anticipated increases in the availability of job control and social support as workers became more aware of the opportunities they had to enact these job characteristics.

Hypothesis 6a: Training workers to enact job control and social support for specific purposes will be associated with increases in job control and social support used for problem-solving, to take a complete break from work and to switch from primary work activities to secondary work activities, the availability of job control, the availability of social support, markers of problem-solving success, well-being and reduced fatigue. Hypothesis 6b: The relationship between training workers to enact job control and social support for specific purposes and fatigue and well-being will be partially mediated by markers of problem-solving success.

\section{Study 2}

\section{Procedure and Sample}

Participants were police officers $(N=15)$ and civilians $(N=13)$ working with vulnerable groups for a police service organization. Fifteen of the sample were female and 13 male. The average age was 43.89 years $(S D=8.09)$. We used a non-equivalent control group design, in which we collected base-line data and follow-up data in control (Total $N=15, N=8$ police, $N=$ 7 civilians) and training groups (Total $N=13, N=7$ police, $N=6$ civilians). Participants were assigned to training or wait list control conditions after consultation with senior officers and administrators on logistically the best locations to deliver the training, given different working patterns. Baseline data were collected 12 weeks before the training and follow-up data were collected approximately five months after the training. 
The training consisted of a single group-based half-day session delivered to three groups of four to seven in a training room at the workplace. Two focus groups with police employees provided information from which the training delivery was adapted according to the aptitudes and knowledge of the participants (Tannenbaum \& Yukl, 1992). The training session was interactive, involving group-based exercises and discussions and focused on enhancing participants' abilities to spot opportunities to use the control workers had in the their jobs and in their social networks better to solve problems at work. The training introduced some formal problem-solving methods in supplementary reading material provided with the training (e.g., fishbone diagrams).

\section{INSERT TABLE 3 HERE}

The main part of the training session was focused around four areas, presented as a visual framework, to assist the participants in forming mental models (Zeitz \& Spoehr, 1989), which may aid retention. Each area comprised several principles, which were presented and discussed in turn, after which participants were asked to conduct a short exercise in pairs or individually, applying the principles to themselves and their work. This method was applied as actively relating the training to workplace behavior enhances learning (Tannenbaum \& Yukl, 1992). The principles and exercises are shown in Table 3. The four areas were: i) Looking for long-term solutions to problems rather than quick fixes; ii) making time and space to approach the problem, work out the best questions to ask about the problem or take a break from the problem; iii) finding the right person to ask about the problem or the right person to be with if a break is needed from solving the problem; iv) reviewing the solution to the problem and sharing any learning. Area ii) of the training specifically related to enacting job control, and in particular for solving problems through enacting job control (CON-PROB) or taking a break from a problem 
either through switching to other work activities (CON-SWITCH TASKS) to facilitate incubation or to take a complete break to get respite from challenging work problems (CONBREAK). Area iii) related specifically to eliciting social support, and again for seeking advice on how to seek problems (SUP-PROB), talking to others about other work issues to facilitate incubation (SUP-SWITCH TASKS) or taking a complete break with other people (SUPBREAK). Those in the training condition were also given the option of participating in up to three group-based top-up sessions with the trainer at one-month intervals after the training session to review progress on the training. All 13 participants completed the three top up sessions.

\section{Measures}

Enacted job control and social support. We adapted the items used in the survey of Study 1, excepting we did not ask participants to rate the items over a given period of time. We used the same rating scale (CON-PROB Time $1 \alpha=.86$, Time $2 \alpha=.78$; SUP-PROB Time $1 \alpha$ $=.81$, Time $2 \alpha=.63 ;$ CON-BREAK Time $1 \alpha=.81$, Time $2 \alpha=.49 ;$ SUP-BREAK Time $1 \alpha$ $=.66$, Time $2 \alpha=.78 ;$ CON-SWITCH TASKS Time $1 \alpha=.70$, Time $2 \alpha=.83$; SUP-SWITCH TASKS Time $1 \alpha=.95$, Time $2 \alpha=.88)$.

Availability of job control and social support. We used the same measures of job control (Time $1 \alpha=.91$, Time $2 \alpha=.94$ ) and social support (Time $1 \alpha=.88$, Time $2 \alpha=.87$ ) as in Study 1, excepting we did not ask participants to rate the availability of job control and social support over a given period of time. We used the same rating scale.

Fatigue and affect. We used the same items and rating scales as the survey in Study 1, but asked participants to rate how they felt at work over the previous month (fatigue Time $1 \alpha$ $=.93$, Time $2 \alpha=.92$; NA Time $1 \alpha=.89$, Time $2 \alpha=.91$; PA Time $1 \alpha=.75$, Time $2 \alpha=.90)$. 
Markers of problem-solving success. We used three markers of problem-solving success. All items were rated on a 5 -point scale $(1=$ never, $5=$ very often $)$. Participants were not asked to rate the items over a given period of time. We used three items each to assess creativity (same items adapted from survey in Study 1 , Time $1 \alpha=.90$, Time $2 \alpha=.86$ ), implementation of ideas (e.g., "How often do you implement new ideas that could improve your work performance?" Time $1 \alpha=.89$, Time $2 \alpha=.81$, Daniels et al., 2011), learning (e.g., "How often do you learn things that help you solve work problems more quickly?" Time $1 \alpha=.79$, Time $2 \alpha=.77$, Daniels et al., 2009). High correlations between the three scales led us to standardized the scale scores and sum them to form a composite (Time $1 \alpha=.82$, Time $2 \alpha=.82$ ).

\section{Analysis}

Hypothesis 6a was examined with analysis of variance (ANOVA) with one betweenparticipants factor (condition: control versus training) and one within-participants factor (time: baseline versus follow-up). Hypothesis 6a would be supported if there is a significant condition*time interaction and the pattern of changes over time shows better outcomes in the training group relative to the control group. Because of the small sample size $(N=28)$, we accepted results as significant at $p<.10$ (two-tailed) to maximize power. Hypothesis $6 \mathrm{~b}$ was tested by using Bayesian estimation methods, which are suitable for examining mediation in small samples (Koopman, Howe, Hollenbeck, \& Sin, 2015). We used multilevel structural equation modelling to model the effect direct effects on fatigue and affect and the indirect effects through markers of problem-solving success of the between-participants condition factor, the within-participants time factor and the condition*time interaction. 


\section{Study 2 Results}

\section{INSERT TABLE 4 HERE}

Table 4 shows the means and standard deviations at baseline and follow-up for the training and control groups. Table 4 also shows two values of Cohen's $d$, representing the effect size for the change in the training group between baseline and follow-up and the effect size for the difference between the changes in training and control groups from baseline to follow-up. The former value of $d$ represents changes in the training group and the latter value represents changes in the training group relative to changes in the control group.

As can be seen, Table 4 shows improvements between baseline and follow-up in the training condition in all variables and in the expected direction. In contrast, most of the variables show deterioration in the control condition, with some variables staying roughly the same. Therefore, the results indicate trends as expected in H6. Using Cohen's (1977) cut-offs for interpreting effect sizes, changes in the training group over time were small in four cases $(d \geq$ $0.20)$, medium in three cases $(d \geq 0.50)$ and large in two cases $(d \geq 0.80)$. Compared to changes in the control group, changes in the training group were small in four cases $(d \geq 0.20)$, medium in one case $(d \geq 0.50)$ and large in seven cases $(d \geq 0.80)$. The larger d's for changes in the training group relative to the control group reflect deterioration in the training group in some indicators.

We found five out of 12 possible interactions between condition and time to be significant at $p<.05$ and a further two at $p<.07$ (all $\mathrm{df} 1 / 26$ ). These were for availability of job control $(F=7.76, p<.01)$, SUP-PROB $(F=5.60, p<.05), \operatorname{CON-BREAK}(F=7.05, p<.05)$, SUP-BREAK $(F=4.60, p<.05)$, CON-SWITCH TASKS $(F=3.67, p<.07)$, problem-solving success $(F=11.66, p<.01)$ and fatigue $(F=3.89, p<.06)$. To interpret these interactions, we 
examined changes from baseline to follow-up for the training and control conditions separately. There was no statistically reliable change in job control in the training condition, but there was a significant deterioration in the control condition $(p<.01)$. There were statistically reliable improvements in the training condition for SUP-PROB $(p<.09)$, CON-BREAK $(p<.05)$, SUP$\operatorname{BREAK}(p<.05)$ and CON-SWITCH TASKS $(p<.06)$ but no statistically reliable changes in the control condition. Problem-solving success improved significantly in the training condition $(p<.06)$ and deteriorated significantly in the control condition $(p<.05)$. There was no statistically reliable change in fatigue in the training condition, but there was a significant increase in fatigue in the control condition $(p<.05)$.

In relation to Hypothesis $6 \mathrm{~b}$, we found that there were statistically reliable indirect effects through problem-solving success of the training intervention on reductions over time in negative affect $(-0.45, p<.05)$ and fatigue $(-0.51, p<.07)$, but there was no significant indirect effect on positive affect $(0.15, n s)$.

\section{Study 2 Discussion}

The results for SUP-PROB, CON-BREAK, SUP-BREAK, CON-SWITCH TASKS and problem-solving success are consistent with H6a, in that we predicted improvements in the enactment of job control and social support for specific purposes to assist in problem-solving. In total, four out of six measures of enacted job control or social support revealed significant improvements consequent to the training. Therefore, the results that are most consistent with H6a are the results most consonant with the focus of the training. Even so, the pattern of means in Table 4 is consistent with trends towards improvements in all variables in the training condition. Moreover, at the time of training delivery, the police service was undergoing significant changes and reduced budgets. It is possible that the results that show deterioration in the control group for 
job control and fatigue, but no change in the training group, reflect a buffering effect of the training on adverse organizational conditions, which is consistent with H6a. Moreover, consistent with $\mathrm{H} 6 \mathrm{~b}$, we found improvements in problem-solving success were associated with reductions in negative affect and fatigue after the training.

\section{General Discussion}

\section{INSERT TABLE 5 HERE}

Table 5 summarizes the results for both studies. In two studies involving three samples, the results show that job design reflects many more processes that can be captured by the usual means of assessing job characteristics by their existence (or not) for a given job at a given point in time. Study 1 shows that the same job characteristic enacted for different purposes can have very a different pattern of relationships with variables than the same job characteristic enacted for a different purposes. Moreover, there does not appear to be equifinality between the enactment of different job characteristics for the same purpose: For any given purpose, there may be different patterns of relationships with other variables depending on whether job control or social support was enacted for that purpose. In Study 2, we demonstrated it is feasible to train workers to enact job characteristics for specific purposes. Both Studies focus on the behaviors of workers enacting their work environment to achieve specific goals. By highlighting this active, behavioral and purposeful nature of job design, our research throws up implications for both the assessment of job characteristics and the design of organizational systems around focal jobs.

In the present research, we focused on a narrow and specific set of goals and only two job characteristics in order to demonstrate the importance of considering what job characteristics are enacted for which purposes. In respect of the assessment of job characteristics, our intention in this paper was not to develop a new set of measures or new approach. The dominant approach to 
assessing job characteristics has the advantage of being underpinned by the availability of multiple instruments with robust psychometric properties, extensive benchmarking data and that can assess an extensive range of job characteristics (cf. Edwards, Webster, Van Laar \& Easton, 2008). A standardized, questionnaire based approach to enacted job characteristics runs into the problems of much greater complexity. For example, workers and their managers may have multiple, conflicting and idiosyncratic goals which may not be possible to know a priori in any given situation. Moreover, job design can be highly contextualized, and the means of operationalizing control over work schedules in one job or for one purpose may be different from how scheduling control could operationalized in another job or for another purpose. The contextualized nature of job design could suggest using assessments that take into account local, organizational contexts (Daniels et al., 2017; Nielsen, Abildgaard \& Daniels, 2014).

To draw on the benefits of both standardized instruments for assessing job characteristics and more context sensitive assessments, we suggest assessments attempt to combine both types of assessment. However, the present research indicates that the context sensitive measurements need to address both what job characteristics workers enact, how that enactment takes place and the purposes of that enactment.

Discerning the purposes of enactment draws attention to how human resource management (HRM) systems may be better configured to encourage desirable outcomes. As the focal point for organizational production, job design has a central role in the delivery of organizational objectives as well as in the safeguarding other stakeholders' interests (e.g., worker well-being, health and safety, Beer, Boselie \& Brewster, 2015). The alignment of job design with HRM practices and also to organizational objectives has received little attention in empirical research (cf. Christina et al., in press). This is despite the well-known Abilities, Motivation and 
Opportunities framework for HRM (Appelbaum et al., 2000), that indicates job design is the means through which workers have the opportunity to use their abilities and motivations to deliver organizational objectives. The idea that job characteristics can be enacted for specific purposes may provide a point of integration for job design, HRM and organizational objectives. For example, to align organizational and worker goals, performance management systems may need to be supplemented with job analysis on whether workers have the opportunity to enact the right job characteristics for optimally achieving those goals and whether the organization has appropriate training or selection procedures in place to ensure workers have the ability to enact the optimal job characteristics for the intended purposes. In this scenario, considering the enactment of job characteristics provides a means of aligning performance management (motivation to enact a specific job characteristic for a specific organizational goal), training, selection processes (abilities to enact a specific job characteristic for a specific organizational goal) and job design (opportunity to enact a specific job characteristic) with organizational goals. Indeed, the training program that was evaluated in Study 2 illustrates how training and development activities can be aligned with job design so that workers can develop skills in using job characteristics in order to solve problems and regulate their own fatigue and well-being.

\section{Limitations and Strengths}

One limitation concerns the relatively small sample sizes for the ESM phase of Study 1 and in Study 2. In relation to the ESM phase of Study 1, ESM studies typically have relatively small sample sizes (see e.g. the special issue edited by Xanthopoulou et al., 2012), and the small sample size is compensated by number of observations and the use of a survey with a larger sample to provide adequate statistical power for the majority of the statistical tests. In relation to Study 2 , in spite of limited statistical power, five out of 12 tests of the training effects yielded 
statistically reliable effects at conventional levels of significance $(p<.05)$ and a further two at $p$ $<.07$. Moreover, all variables trended in the hypothesized direction and estimates of effect sizes indicated some substantial effects of the training intervention. Notwithstanding, a more powerful design with repeated follow-up assessments would have been able to detect more subtle, shortterm, longer-term, conditional or accumulative effects of the training (cf. Schmitz \& Wiese, 2006). Related to sample size is the low response rate from one organization in the survey phase of Study 1 .

Common method variance (CMV) is a potential problem because we used self-report measures in Study 1, although workers are arguably best placed to know their own behaviors, reasons for those behaviors, affect, and whether ideas add value to their work. However, CMV may be mitigated by the greater recall accuracy afforded by ESM (Study 1, Sample 1, Bolger, Davis \& Rafaeli, 2003) and variation in response formats (Study 1, Samples 1 and 2, Podsakoff, MacKenzie \& Podsakoff, 2012).

The strengths of the present studies are the use of three samples and three different methods. Demonstrating generalizable findings across two samples in Study 1 addresses issues concerned with the limitations of a single data set and methodology, including a low response rate in part of one sample and a small sample size in another. The general principles supported in Study 1 and Study 2 are strengthened by the use of a quasi-experimental design in Study 2, which also obviates problems of CMV.

\section{Conclusion}

In the present research, we have illustrated the importance for research and assessment and guidance on job design to go beyond simply assessing the presence or absence of job characteristics. The present research indicates that how workers use the characteristics of their 
jobs can lead to different outcomes depending on the goals they are pursuing. However, the behavioral and teleological bases of enacted job characteristics does suggest that workers can learn to use their job characteristics more effectively for their own and/or organizational benefits. Therefore, the results suggest it is useful for job redesign interventions to improve well-being, health and safety to be supplemented with employment practices that ensure workers have the motivation and abilities to use the features of redesigned work in ways that enhance well-being, health and safety. 


\section{References}

Appelbaum, E.M., Bailey, T., Berg, P., \& Kalleberg, A. (2000) Manufacturing competitive advantage: the effects of high performance work systems on plant performance and company outcomes. Ithaca, NY: Cornell University Press.

Bakker, A.B., \& Demerouti, E. (2007). The job demands-resources model: State of the art. Journal of Managerial Psychology, 22, 309-328. DOI:10.1108/02683940710733115.

Bakker, A., Daniels, K. (eds) (2012). A day in the life of a happy worker. London: Psychology Press.

Beer, M., Boselie, P., \& Brewster, C. (2015). Back to the future: Implications for the field of HRM of the multistakeholder perspective proposed 30 years ago. Human Resource Management, 54, 427-438. DOI:10.1002/hrm.21726.

Bolger, N., Davis, A., \& Rafaeli, E. (2003). Diary methods: Capturing life as it is lived. Annual Review of Psychology, 54, 579-616. DOI:10.1146/annurev.psych.54.101601.145030.

Breaugh, J.A. (1985). The measurement of work autonomy. Human Relations, 38, 551-570.

Cherns, A. (1987). Principles of sociotechnical design revisited. Human Relations, 40, 153-161. DOI:10.1177/001872678704000303.

Christina, S., Dainty, A., Daniels, K., Tregaskis, O., Waterson, P. (in press). Shut the fridge door! HRM alignment, job redesign and energy performance. Human Resource Management Journal. DOI: 10.1111/1748-8583.12144.

Clegg, C. W. (2000). Sociotechnical principles for system design. Applied ergonomics, 31, 463477. DOI: 10.1016/S0003-6870(00)00009-0.

Cohen, J. (1977). Statistical power analysis for the behavioral sciences. New York: Academic Press. 
Combs, J., Liu, Y., Hall, A. \& Ketchen, D. (2006). How much do high-performance work practices matter? A meta-analysis of their effects on organizational performance. Personnel Psychology, 59, 501-528. DOI: 10.1111/j.1744-6570.2006.00045.x.

Cousins, R., Mackay, C.J., Clarke, S.D., Kelly, C., Kelly, P.J., \& McCaig, R.H. (2004). 'Management standards' work-related stress in the UK: Practical development. Work \& Stress, 18, 113-136. DOI: 10.1080/02678370410001734322.

Daniels, K. (2000). Measures of five aspects of affective well-being at work. Human Relations, 53, 275-294. DOI: $10.1177 / \mathrm{a} 010564$.

Daniels, K. (2006). Rethinking job characteristics in work stress research. Human Relations, 59, 267-290. DOI: 10.1177/0018726706064171.

Daniels, K. (2011). Stress and well-being are still issues and something still needs to be done: Or why agency and interpretation are important for policy and practice. In G.P. Hodgkinson and J.K. Ford (Eds.), International review of industrial and organizational psychology volume 25 (pp 1-45). New York: Wiley.

Daniels, K., (2012). Jobs characteristics and problem-solving (pp 58-71). In Bakker, A. and Daniels, K. (eds.) A Day in the Life of A Happy Worker. London: Psychology Press.

Daniels, K., Beesley, N.J., Cheyne, A.J.T., Wimalasiri, V.P. (2008). Coping processes linking the Demands-Control-Support model, affect, and risky decisions at work. Human Relations, 61, 845-874. DOI:10.1177/0018726708093543.

Daniels, K., Beesley, N.J., Wimalasiri, V.P., \& Cheyne, A.J.T. (2013a). Problem-solving and well-being: Exploring the instrumental role of job control and social support. Journal of Management, 39, 1016-1043. DOI:10.1177/0149206311430262. 
Daniels, K., Boocock, G., Glover, J., Hartley, R., \& Holland, J. (2009). An experience sampling study of learning, affect, and the Demands Control Support model. Journal of Applied Psychology, 94, 1003-1017. DOI:10.1037/a0015517.

Daniels, K., Gedikli, C., Watson, D., Semkina, A., Vaughn, O. (2017). Job design, employment practices and well-being: A systematic review of intervention studies. Ergonomics, 60, 1177-1196. DOI:10.1080/00140139.2017.1303085.

Daniels, K., Glover J., Beesley, N., Wimalasiri, V., Cohen, L., Cheyne, A., \& Hislop D. (2013b). Utilizing job resources: Qualitative evidence of the roles of job control and social support in problem solving. Work \& Stress, 27, 200-221. DOI:10.1080/02678373.2013.792471.

Daniels, K., Glover, J., \& Mellor, N. (2014). An experience sampling study of expressing affect, daily affective well-being, relationship quality and perceived performance. Journal of Occupational and Organizational Psychology, 87, 781-805. DOI:10.1111/joop.12074.

Daniels, K., Harris, C. (2005). A daily diary study of coping in the context of the job demandscontrol-support model. Journal of Vocational Behavior, 66, 219-237. DOI: 10.1016/j.jvb.2004.10.004.

Daniels, K., Hartley, R., Travers, C. (2006). Beliefs about stressors alter stressors’ impact: evidence from two experience-sampling studies. Human Relations, 59, 1261-1285. DOI: $10.1177 / 0018726706069768$.

Daniels, K., Wimalasiri, V.P., Cheyne, A.J.T., \& Story, V. (2011). Linking the DemandsControl-Support Model to innovation: The moderating role of personal initiative on the generation and implementation of ideas. Journal of Occupational and Organizational Psychology. 84, 581-598. DOI:10.1348/096317910X494269. 
Demerouti, E., Xanthopoulou, D., Petrou, P., \& Karagkounis, C. (2017). Does job crafting assist dealing with organizational changes due to austerity measures? Two studies among Greek employees. European Journal of Work and Organizational Psychology, 26, 574589. DOI:10.1080/1359432X.2017.132587.

Dollard, M., Skinner, N., Tuckey, M.R. \& Bailey, T. (2007). National surveillance of psychosocial risk factors in the workplace: An international overview. Work \& Stress, 21, 1-29. DOI:10.1080/02678370701254082.

Elsbach, K.D., \& Hargadon, A.B. (2006). Enhancing creativity through “mindless" work: A framework of workday design. Organization Science, 17, 470-483. DOI: 10.1287/orsc.1060.0193.

Feldman, M.S. \& Worline, M.C. (2011). Resourcefulness: Resources, resourcing, and amplicative cycles in organizations. In K.S. Cameron \& G.M. Spreitzer (Eds.), Oxford handbook of positive organizational scholarship (pp 629-641). Oxford: Oxford University Press.

Fritz, C., Lam, C.F., \& Spreitzer, G.M. (2011). It's the little things that matter: An examination of knowledge workers' energy management. Academy of Management Perspectives, 25, 28-39.

George, J.M. (2007). Creativity in organizations. Academy of Management Annals, 1, 439-477. DOI:10.1080/078559814.

Grant, A.M., \& Parker, S.K. (2009). Redesigning work design theories: The rise of relational and proactive perspectives. Academy of Management Annals, 3, 317-375.

DOI:10.1080/19416520903047327. 
Griffin, M.A., Neal, A., \& Parker, S.K. (2007). A new model of work role performance: Positive behavior in uncertain and interdependent contexts. Academy of Management Journal, 50, 327-347. DOI: 10.5465/AMJ.2007.24634438.

Hackman, J. R., \& Oldham, G. R. (1975). Development of the job diagnostic survey. Journal of Applied psychology, 60, 159-170. DOI:10.1037/h0076546.

Hackman, J.R., \& Oldham, G.R. (1976). Motivation through the design of work: Test of a theory. Organizational Behavior and Human Performance, 16, 250-279. DOI: 10.1016/0030-5073(76)90016-7.

Hox, J.J., Maas, C.J., \& Brinkhuis, M.J. (2010). The effect of estimation method and sample size in multilevel structural equation modeling. Statistica Neerlandica, 64, 157-170. DOI:10.1111/j.1467-9574.2009.00445.x.

Humphrey, S. E., Nahrgang, J. D., \& Morgeson, F. P. (2007). Integrating motivational, social, and contextual work design features: a meta-analytic summary and theoretical extension of the work design literature. Journal of Applied Psychology, 92, 1332-1356. DOI:10.1037/0021-9010.92.5.1332.

Karasek, R.A., \& Theorell, T. (1990). Healthy work. New York: Basic Books.

Karasek, R., Brisson, C., Kawakami, N., Houtman, I., Bongers, P., \& Amick, B. (1998). The Job Content Questionnaire (JCQ): an instrument for internationally comparative assessments of psychosocial job characteristics. Journal of Occupational Health Psychology, 3, 322. DOI:10.1037/1076-8998.3.4.322.

Koopman, J., Howe, M., Hollenbeck, J. R., \& Sin, H. P. (2015). Small sample mediation testing: misplaced confidence in bootstrapped confidence intervals. Journal of Applied Psychology, 100, 194-202. DOI:10.1037/a0036635. 
Kristensen, T. S., Hannerz, H., Høgh, A., \& Borg, V. (2005). The Copenhagen Psychosocial Questionnaire - A tool for the assessment and improvement of the psychosocial work environment. Scandinavian Journal of Work, Environment \& Health, 31, 438-449. DOI: 10.5271/sjweh.948.

Lee, I. A., \& Preacher, K. J. (2013, September). Calculation for the test of the difference between two dependent correlations with one variable in common [Computer software]. Available from http://quantpsy.org.

Miner, A., Glomb, T., \& Hulin, C. (2005). Experience sampling mood and its correlates at work. Journal of Occupational and Organizational Psychology, 78, 171-193. DOI: $10.1348 / 096317905 X 40105$.

Morgeson, F. P., \& Humphrey, S. E. (2006). The Work Design Questionnaire (WDQ): developing and validating a comprehensive measure for assessing job design and the nature of work. Journal of Applied Psychology, 91, 1321-1339. DOI: 10.1037/00219010.91.6.1321.

Morgeson, F. P., \& Humphrey, S. E. (2008). Job and team design: Toward a more integrative conceptualization of work design. Research in Personnel and Human Resources Management, 27, 39-91. DOI:10.1016/S0742-7301(08)27002-7.

Muthén, B. (2010). Bayesian analysis in Mplus: A brief introduction. https://www.statmodel.com/download/IntroBayesVersion\%203.pdf

Nahrgang, J.D., Morgeson, F.P. \& Hofmann, D.A. (2011). Safety at work: A meta-analytic investigation of the link between job demands, job resources, burnout, engagement, and safety outcomes. Journal of Applied Psychology, 96, 71-94. DOI:10.1037/a0021484. 
Nielsen, K., Abildgaard, J.S., Daniels, K. (2014). Putting context into organizational intervention design: Using tailored questionnaires to measure initiatives for worker well-being. Human Relations, 67, 1537-1560. DOI:10.1177/0018726714525974.

Nieuwenhuijsen, K., Bruinvels, D., \& Frings-Dresen, M. (2010). Psychosocial work environment and stress-related disorders, a systematic review. Occupational Medicine, 60, 277-286. DOI: $10.1093 /$ occmed/kqq081.

Oldham, G.R., \& Rotchford, N.L. (1983). Relationships between office characteristics and employee reactions: A study of the physical environment. Administrative Science Quarterly, 28, 542-556. DOI:10.2307/2393008.

Parker, S. K., \& Ohly, S. (2008). Designing motivating work. In R. Kanfer, G. Chen, \& R. Pritchard (Eds.), Work motivation: Past, present and future (pp. 233-384). New York: Routledge.

Peterson, M.F. (1998). Embedded organizational events: the units of process in organizational science. Organization Science, 9, 16-33. DOI: 10.1287/orsc.9.1.16.

Podsakoff, P.M., MacKenzie, S.B., \& Podsakoff, N.P. (2012). Sources of method bias in social science research and recommendations on how to control it. Annual Review of Psychology, 63, 539-569. DOI:10.1146/annurev-psych-120710-100452.

Rogelberg, S.G. \& Stanton, J.M. (2007). Introduction understanding and dealing with organizational survey nonresponse. Organizational Research Methods, 10: 195-209. DOI: $10.1177 / 1094428106294693$.

Schmitz, B., \& Wiese, B. S. (2006). New perspectives for the evaluation of training sessions in self-regulated learning: Time-series analyses of diary data. Contemporary Educational Psychology, 31, 64-96. DOI: 10.1016/j.cedpsych.2005.02.002. 
Scott, S.G., \& Bruce, R.A. (1994). Determinants of innovative behavior: A path model of individual innovation in the workplace. Academy of Management Journal, 37, 580-607. DOI:10.2307/256701.

Sio, U.N., \& Ormerod, T.C. (2009). Does incubation enhance problem solving? A meta-analytic review. Psychological Bulletin, 135, 94-120. DOI:10.1037/a0014212.

Skinner, E.A., Edge, K., Altman, J., \& Sherwood, H. (2003). Searching for the structure of coping: a review and critique of category systems for classifying ways of coping. Psychological Bulletin, 129, 216-269. DOI:10.1037/0033-2909.129.2.216.

Sonnentag, S., \& Fritz, C. (2015). Recovery from job stress: The stressor-detachment model as an integrative framework. Journal of Organizational Behavior, 36, S72-S103. DOI: 10.1002/job.1924.

Stansfeld, S., \& Candy, B. (2006). Psychosocial work environment and mental health-a metaanalytic review. Scandinavian Journal of Work, Environment \& Health, 32, 443-462. DOI:10.5271/sjweh.1050.

Strauss, K., Griffin, M. A., Parker, S. K., \& Mason, C. M. (2015). Building and sustaining proactive behaviors: The role of adaptivity and job satisfaction. Journal of Business and Psychology, 30, 63-72. DOI: 10.1007/s10869-013-9334-5.

Tannenbaum, S. I., \& Yukl, G. (1992). Training and development in work organizations. Annual Review of Psychology, 43, 399-441. DOI:10.1146/annualrev.ps43.020192.002151.

Theorell, T., Hammarström, A., Aronsson, G., Träskman Bendz, L., Grape, T., Hogstedt, C., Marteinsdottir, I., Skoog, I., \& Hall, C. (2015). A systematic review including metaanalysis of work environment and depressive symptoms. BMC Public Health 15, no.1. DOI:10.1186/s12889-015-1954-4. 
Van den Broeck, A., Vansteenkiste, M., De Witte, H., \& Lens, W. (2008). Explaining the relationships between job characteristics, burnout, and engagement: The role of basic psychological need satisfaction. Work \& Stress, 22, 277_294. DOI: $10.1080 / 02678370802393672$.

van den Heuvel, M., Demerouti, E., \& Peeters, M.C.W. (2015). The job crafting intervention: Effects on job resources, self-efficacy, and affective well-being. Journal of Occupational and Organizational Psychology, 88, 511-532. DOI: 10.1111/joop.12128.

Van Wingerden, J., Derks, D., \& Bakker, A.B. (2015). The impact of personal resources and job crafting interventions on work engagement and performance. Human Resource Management. DOI: 10.1002/hrm.21758.

Warr, P. (2007). Work, happiness and unhappiness. Mahwah, NJ: Erlbaum.

Watson, D., \& Tellegen, A. (1985). Toward a consensual structure of mood. Psychological Bulletin, 98, 219-235. DOI:10.1037/0033-2909.98.2.219.

Williams, L. J., Vandenberg, R. J., \& Edwards, J. R. (2009). 12 structural equation modeling in management research: a guide for improved analysis. Academy of Management Annals, 3, 543-604. DOI:10.1080/19416520903065683.

Wrzesniewski, A., \& Dutton, J. (2001). Crafting a job: Revisioning employees as active crafters of their work. Academy of Management Review, 26, 179-201. DOI: 10.2307/259118.

Xanthopoulou, D., Bakker, A.B., \& Ilies, R. (2012). Everyday working life: Explaining withinperson fluctuations in employee well-being. Human Relations, 65, 1051-1069. DOI: $10.1177 / 0018726712451283$.

Zeitz, C. M., \& Spoehr, K. T. (1989). Knowledge organization and the acquisition of procedural expertise. Applied Cognitive Psychology, 3, 313-336. DOI:10.1002/acp.2350030404. 
Table 1. Study 1: Means, standard deviations, internal consistencies and correlations for ESM data.

\begin{tabular}{|c|c|c|c|c|c|c|c|c|c|c|c|c|c|c|c|}
\hline & $M$ & $S D$ & 1 & 2 & 3 & 4 & 5 & 6 & 7 & 8 & 9 & 10 & 11 & 12 & 13 \\
\hline 1. (Availability of) job control & 3.69 & .53 & .71 & $.26^{*}$ & .19 & .03 & .06 & .01 & .19 & $.28^{*}$ & .16 & .16 & -.06 & -.22 & .20 \\
\hline 2. (Availability of) social support & 4.22 & .53 & -- & .69 & -.19 & .13 & -.18 & .01 & -.17 & .11 & .01 & .00 & .10 & -.18 & .16 \\
\hline 3. Job control for problem-solving (CON-PROB) & 2.36 & 1.49 & -- & -- & .84 & .23 & $.44^{* *}$ & .15 & $.64^{* *}$ & $.27^{*}$ & .16 & .17 & .00 & .12 & -.07 \\
\hline 4. Social support for problem-solving (SUP-PROB) & 2.68 & 1.74 & -- & -- & $.38^{* *}$ & .88 & .12 & .15 & .05 & $.27^{*}$ & $.24^{*}$ & .14 & .02 & -.19 & .16 \\
\hline 5. Job control for a complete break (CON-BREAK) & 1.86 & 1.16 & -- & -- & $.15^{* *}$ & .01 & .76 & $.61^{* *}$ & $.59^{* *}$ & $.41^{* *}$ & -.05 & -.03 & -.01 & .08 & -.17 \\
\hline $\begin{array}{l}\text { 6. Talking to others for a complete break (SUP- } \\
\text { BREAK) }\end{array}$ & 2.25 & 1.29 & -- & -- & -.02 & .02 & $.51^{* *}$ & .81 & $.24^{*}$ & $.36^{* *}$ & $-.28^{*}$ & -.21 & -.02 & -.11 & .04 \\
\hline $\begin{array}{l}\text { 7. Job control to switch to secondary tasks (CON- } \\
\text { SWITCH TASKS) }\end{array}$ & 2.29 & 1.26 & -- & -- & $.50^{* *}$ & $.12^{* *}$ & $.26^{* *}$ & $.22^{* *}$ & .80 & $.64^{* *}$ & .12 & .16 & .11 & .10 & -.17 \\
\hline $\begin{array}{l}\text { 8. Talking about secondary tasks (SUP-SWITCH } \\
\text { TASKS) }\end{array}$ & 2.55 & 1.21 & -- & -- & $.31^{* *}$ & $.18^{* *}$ & $.21^{* *}$ & $.31^{* *}$ & $.64^{* *}$ & .78 & .19 & .20 & .11 & -.05 & .11 \\
\hline 9. Problem-solving demands & 1.33 & 1.38 & -- & -- & $.28^{* *}$ & $.28^{* *}$ & .00 & -.07 & $.11^{* *}$ & .07 & -- & $.63^{* *}$ & .13 & .09 & .17 \\
\hline 10. Creativity & 1.63 & 0.83 & -- & -- & $.17^{* *}$ & $.28^{* *}$ & .04 & .05 & $.14^{* *}$ & $.18^{* *}$ & $.33^{* *}$ & .87 & -.05 & -.08 & $.34^{* *}$ \\
\hline 11. Fatigue & 2.34 & 1.12 & -- & -- & -.04 & -.04 & .00 & $-.09^{*}$ & .04 & -.02 & .03 & .01 & .90 & $.54^{* *}$ & $-.39^{* *}$ \\
\hline 12. Anxious negative affect (NA) & 1.82 & 0.91 & -- & -- & $.26^{* *}$ & $.13^{* *}$ & .04 & $-.09^{*}$ & $.15^{* *}$ & $.10^{* * *}$ & $.13^{* *}$ & -.01 & $.24^{* *}$ & .85 & $-.44^{* *}$ \\
\hline 13. Positive affect (PA) & 3.22 & 0.96 & -- & -- & -.06 & $.18^{* *}$ & .03 & $.10^{* *}$ & -.01 & .05 & -.02 & $.22^{* *}$ & $-.43^{* * *}$ & $-.31^{* *}$ & .88 \\
\hline
\end{tabular}

Note. $\quad N=71$, no. observations $=693$ for most within-person correlations, 463 for correlations for CON-PROB and SUP-PROB.

Correlations involving CON-PROB and SUP-PROB based on data where a problem had been reported in the previous hour.

Correlations below the primary diagonal are based on variables centered at each participant's mean. Reliabilities (Cronbach's alpha) shown on primary diagonal. Reliabilities for ESM data based on hourly observations. Correlations above the primary diagonal are between-person correlations based each individual's mean. ${ }^{*} p<.05,{ }^{* *} p<.01$. 
Table 2. Study 1: Means, standard deviations, internal consistencies and correlations for survey data.

\begin{tabular}{|c|c|c|c|c|c|c|c|c|c|c|c|c|c|c|c|}
\hline & $M$ & $S D$ & 1 & 2 & 3 & 4 & 5 & 6 & 7 & 8 & 9 & 10 & 11 & 12 & 13 \\
\hline 1. (Availability of) job control & 3.83 & 0.68 & .82 & & & & & & & & & & & & \\
\hline 2. (Availability of) social support & 3.81 & 0.79 & $.38^{* *}$ & .80 & & & & & & & & & & & \\
\hline 3. Job control for problem-solving (CON-PROB) & 3.49 & 0.91 & $.18^{* *}$ & $.16^{* *}$ & .77 & & & & & & & & & & \\
\hline 4. Social support for problem-solving (SUP-PROB) & 3.64 & 0.89 & $.13^{*}$ & $.46^{* *}$ & $.33^{* *}$ & .78 & & & & & & & & & \\
\hline 5. Job control for a complete break (CON-BREAK) & 2.51 & 1.13 & $.28^{* *}$ & $.16^{* *}$ & $.27^{* *}$ & $.17^{* *}$ & .82 & & & & & & & & \\
\hline $\begin{array}{l}\text { 6. Talking to others for a complete break (SUP- } \\
\text { BREAK) }\end{array}$ & 3.00 & 1.02 & $.14^{*}$ & $.20^{* *}$ & .03 & $.25^{* *}$ & $.46^{* *}$ & .75 & & & & & & & \\
\hline $\begin{array}{l}\text { 7. Job control to switch to secondary tasks (CON- } \\
\text { SWITCH TASKS) }\end{array}$ & 2.71 & 1.00 & $.15^{*}$ & .11 & $.31^{* *}$ & $.20^{* *}$ & $.52^{* *}$ & $.41^{* *}$ & .82 & & & & & & \\
\hline $\begin{array}{l}\text { 8. Talking about secondary tasks (SUP-SWITCH } \\
\text { TASKS) }\end{array}$ & 3.09 & 0.94 & .06 & $.22^{* *}$ & $.12^{*}$ & $.32^{* *}$ & $.34^{* *}$ & $.61^{* *}$ & $.61^{* *}$ & .86 & & & & & \\
\hline 9. Problem-solving demands & 3.42 & 0.83 & .10 & -.07 & $.47^{* *}$ & $.22^{* *}$ & $.20^{* *}$ & .04 & $.23^{* *}$ & .07 & .80 & & & & \\
\hline 10. Creativity & 3.11 & 0.91 & .03 & .03 & $.30^{* *}$ & -.03 & $.20^{* * *}$ & .03 & $.18^{* *}$ & .07 & $.38^{* *}$ & .90 & & & \\
\hline 11. Fatigue & 2.79 & 1.06 & $-.23^{* *}$ & $-.18^{* *}$ & .01 & -.03 & .00 & .09 & $.20^{* *}$ & $.17^{* *}$ & $.12^{*}$ & .00 & .86 & & \\
\hline 12. Anxious negative affect (NA) & 2.57 & 1.09 & $-.24^{* *}$ & $-.19^{* *}$ & $.14^{*}$ & .02 & .01 & -.01 & $.15^{* *}$ & .04 & $.23^{* *}$ & .08 & $.55^{* *}$ & .89 & \\
\hline 13. Positive affect (PA) & 3.58 & 1.12 & $.28^{* * *}$ & $.29^{* *}$ & $.18^{* *}$ & $.13^{*}$ & .05 & $-.14^{* * *}$ & -.02 & -.05 & .09 & $.33^{* *}$ & $-.36^{* *}$ & $-.32^{* * *}$ & .88 \\
\hline
\end{tabular}

Note. $\quad N=299$.

Reliabilities (Cronbach's alpha) shown on primary diagonal.

${ }^{*} p<.05,{ }^{* *} p<.01$. 
Table 3. Overview of training areas, principles and exercises.

\begin{tabular}{|c|c|c|}
\hline Area & The principles & Exercise \\
\hline $\begin{array}{l}\text { 1. Looking for long- } \\
\text { term solutions to } \\
\text { problems rather than } \\
\text { quick fixes }\end{array}$ & $\begin{array}{l}\text { First solution not necessarily best solution } \\
\text { Think about finding the optimal solution } \\
\text { Introduction to the method of analyzing hierarchy of } \\
\text { problem and identifying barriers and enablers }\end{array}$ & $\begin{array}{l}\text { Identify a problem. } \\
\text { 1. What makes it a problem for you and why? } \\
\text { 2. Break it down into the sub-problems (5 } \\
\text { whys may be useful technique) } \\
\text { 3. Identify which sub-problems you could } \\
\text { solve and which you could not. Why? }\end{array}$ \\
\hline $\begin{array}{l}\text { 2. Making time and } \\
\text { space to approach the } \\
\text { problem, work out the } \\
\text { best questions to ask } \\
\text { about the problem or } \\
\text { take a break from the } \\
\text { problem }\end{array}$ & $\begin{array}{l}\text { Time is very important. } \\
\text { Micro-breaks and how long is the optimal break. } \\
\text { Unscheduled breaks, scheduled breaks; Incubation, } \\
\text { recovery, reflection } \\
\text { Knowing when to take a break } \\
\text { Finding a good time: store problems } \\
\text { How to use it - go to others to find best solution, } \\
\text { switch tasks, take a break }\end{array}$ & $\begin{array}{l}\text { 1. Do you have any time-habits? What are } \\
\text { they? } \\
\text { 2. What opportunities do you have to take a } \\
\text { break? } \\
\text { 3. How much time would you need? } \\
\text { 4. What new time habits could you introduce? }\end{array}$ \\
\hline $\begin{array}{l}\text { 3. Finding the right } \\
\text { person to ask about } \\
\text { the problem or the } \\
\text { right person to be with } \\
\text { if a break is needed } \\
\text { from solving the } \\
\text { problem }\end{array}$ & $\begin{array}{l}\text { People are important: support, advice, learning } \\
\text { Other reasons? Finding the best solution? Part of a } \\
\text { problem? }\end{array}$ & $\begin{array}{l}\text { Complete your personal network chart } \\
\text { 1. Who do you go to for advice or support } \\
\text { with problems? } \\
\text { 2. Think about your regular challenges and } \\
\text { problems, who would you like to go to for } \\
\text { advice? } \\
\text { 3. What, if anything is stopping you? }\end{array}$ \\
\hline $\begin{array}{l}\text { 4. Reviewing the } \\
\text { solution to the } \\
\text { problem and sharing } \\
\text { any learning. }\end{array}$ & $\begin{array}{l}\text { Take the time and space to review how problems } \\
\text { were solved/not solved. } \\
\text { Would a different solution have worked better in the } \\
\text { long term? } \\
\text { Would your solution work for colleagues' problems? } \\
\text { What means could you use to share learning? }\end{array}$ & $\begin{array}{l}\text { Think about a recurring problem you have tackled } \\
\text { 1. Did your solution work? Why/why not; } \\
\text { what could you do better/differently next } \\
\text { time? } \\
\text { 2. What means could you use to share what } \\
\text { you've learned and find out what others } \\
\text { have learned? } \\
\text { 3. Is there anything else you could do? }\end{array}$ \\
\hline
\end{tabular}


Table 4. Study 2: Means, standard deviations and Cohen's $d$ at baseline and follow-up for training and control conditions

\begin{tabular}{|c|c|c|c|c|c|c|}
\hline & $\begin{array}{l}\text { Training } \\
\text { baseline } \\
\text { Mean }(S D)\end{array}$ & $\begin{array}{l}\text { Training } \\
\text { follow-up } \\
\text { Mean }(S D)\end{array}$ & $\begin{array}{l}\text { Control } \\
\text { baseline } \\
\text { Mean }(S D)\end{array}$ & $\begin{array}{l}\text { Control } \\
\text { follow-up } \\
\text { Mean }(S D)\end{array}$ & $\begin{array}{l}d \\
\Delta \mathrm{TB} \text { to } \mathrm{TFU}\end{array}$ & $\begin{array}{l}l d \\
\Delta(\mathrm{TB}-\mathrm{TFU}) \\
\text { to } \\
\Delta(\mathrm{CB}-\mathrm{CFU})\end{array}$ \\
\hline (Availability of) job control & $3.46(0.75)$ & $3.53(0.77)$ & $3.42(0.84)$ & $2.90(0.70)$ & 0.09 & 1.25 \\
\hline (Availability of) social support & $3.98(0.79)$ & $4.19(0.61)$ & $3.77(0.93)$ & $3.58(0.83)$ & 0.30 & 0.58 \\
\hline Job control for problem-solving (CON-PROB) & $3.73(0.56)$ & $4.04(0.61)$ & $3.80(0.92)$ & $3.77(0.82)$ & 0.53 & 0.46 \\
\hline Social support for problem-solving (SUP-PROB) & $3.85(0.77)$ & $4.08(0.64)$ & $3.87(0.88)$ & $3.50(0.78)$ & 0.32 & 1.02 \\
\hline Job control for a complete break (CON-BREAK) & $2.08(1.06)$ & $2.65(0.94)$ & $2.73(0.98)$ & $2.50(0.46)$ & 0.57 & 1.17 \\
\hline Talking to others for a complete break (SUP-BREAK) & $2.65(0.77)$ & $3.31(0.85)$ & $2.97(1.04)$ & $2.80(0.84)$ & 0.81 & 0.92 \\
\hline $\begin{array}{l}\text { Job control to switch to secondary tasks (CON-SWITCH } \\
\text { TASKS) }\end{array}$ & $2.23(0.67)$ & $2.81(0.72)$ & $2.53(1.19)$ & $2.30(0.65)$ & 0.83 & 0.80 \\
\hline Talking about secondary tasks (SUP-SWITCH TASKS) & $2.92(0.84)$ & $3.12(0.87)$ & $3.13(1.26)$ & $2.90(0.69)$ & 0.23 & 0.35 \\
\hline Problem-solving success & $-0.37(0.71)$ & $0.07(0.80)$ & $0.34(0.96)$ & $-0.07(0.98)$ & 0.58 & 1.63 \\
\hline Fatigue & $3.31(1.18)$ & $3.12(0.92)$ & $2.71(1.16)$ & $3.40(1.50)$ & 0.18 & 0.83 \\
\hline Anxious negative affect (NA) & $2.74(0.81)$ & $2.56(0.75)$ & $2.58(1.38)$ & $2.57(1.43)$ & 0.23 & 0.26 \\
\hline Positive affect (PA) & $3.49(0.81)$ & $3.56(0.99)$ & $4.02(1.01)$ & $3.67(1.37)$ & 0.08 & 0.42 \\
\hline
\end{tabular}

Note. $\quad N=28$.

$\mathrm{TB}=$ training group score at baseline, $\mathrm{TFU}=$ training group score at follow-up, $\mathrm{CB}=$ control group score at baseline, $\mathrm{CFU}=\mathrm{control}$ group score at follow-up 
Table 5. Summary of results for hypotheses and research question for studies 1 and 2.

Results

H1 1a: The following are separate constructs: i) job control used for problem-solving (CON-PROB); ii) job control Fully supported used to take a complete break from work (CON-BREAK); iii) job control used to switch from primary work activities to secondary work activities (CON-SWITCH TASKS); iv) social support used for problem-solving (SUP-PROB); v) social support used to take a complete break from work (SUP-BREAK); vi) social support used to switch from primary work activities to secondary work activities (SUP-SWITCH TASKS).

$1 \mathrm{~b}$ : The presence of a) job control and b) social support are distinct constructs from i) job control used for problem-solving (CON-PROB); ii) job control used to take a complete break from work (CON-BREAK); iii) job control used to switch from primary work activities to secondary work activities (CON-SWITCH TASKS); iv) social support used for problem-solving (SUP-PROB); v) social support used to take a complete break from work (SUP-BREAK); vi) social support used to switch from primary work activities to secondary work activities (SUP-SWITCH TASKS).

H2 2a: Compared to the presence of social support, the presence of job control is more closely related to i) job control used for problem-solving (CON-PROB); ii) job control used to take a complete break from work (CON-BREAK); and iii) job control used to switch from primary work activities to secondary work activities (CON-SWITCH TASKS).

2b: Compared to the presence of job control, the presence of social support job control is more closely related to i) social support used for problem-solving (SUP-PROB); ii) social support used to take a complete break from work (SUP-BREAK); iii) social support used to switch from primary work activities to secondary work activities (SUP-SWITCH TASKS).

\footnotetext{
H3 3a: Job control used for problem-solving (CON-PROB) is more closely related to social support used for problem-solving (SUP-PROB) than i) social support used to take a complete break from work (SUP-BREAK) or ii) social support used to switch from primary work activities to secondary work activities (SUP-SWITCH TASKS).

3b: Job control used to take a complete break (CON-BREAK) is more closely related to social support to take a Some support complete break (SUP-BREAK) than i) social support used for problem-solving (SUP-PROB) or ii) social support used to switch from primary work activities to secondary work activities (SUP-SWITCH TASKS). 
Table 5. Continued.

H3 3c: Job control used to switch from primary work activities to secondary work activities (CON-SWITCH TASKS) is more closely related to social support to switch from primary work activities to secondary work

Fully supported activities (SUP-SWITCH TASKS) than i) social support used for problem-solving (SUP-PROB) and ii) social support used to take a complete break from work (SUP-BREAK).

H4 4a: Problem-solving demands are correlated with i) job control used for problem-solving (CON-PROB) and ii) social support used for problem-solving (SUP-PROB).

4b: Creativity is correlated with i) job control used for problem-solving (CON-PROB); ii) job control used to switch from primary work activities to secondary work activities (CON-SWITCH TASKS); iii) social support used for problem-solving (SUP-PROB); and iv) social support used to switch from primary work activities to secondary work activities (SUP-SWITCH TASKS).

$4 \mathrm{c}$ : Poor well-being and fatigue are negatively related to i) job control used to take a complete break from work (CON-BREAK) and ii) social support used to take a complete break from work (SUP-BREAK).

4d: Poor well-being and fatigue are positively related to i) job control used to switch from primary work activities to secondary work activities (CON-SWITCH TASKS) and ii) social support used to switch from primary work activities to secondary work activities (SUP-SWITCH TASKS).

4e: Correlations between a range of other variables and the availability of job control are different from correlations between those same variables and either CON-PROB, CON-BREAK or CON-SWITCH TASKS.

4f: Correlations between a range of other variables and the availability of social support are different from correlations between those same variables and either SUP-PROB, SUP-BREAK or SUP-SWITCH TASKS.

Some support

Some support

5: Are relationships with other variables different for job control enacted for specific purposes and social support enacted for specific purposes?

H6 6a: Training workers to enact job control and social support for specific purposes will be associated with increases in job control and social support used for problem-solving, to take a complete break from work and to switch from primary work activities to secondary work activities, the availability of job control, the availability of social support, markers of problem-solving success, well-being and reduced fatigue.

6b: The relationship between training workers to enact job control and social support for specific purposes and fatigue and well-being will be partially mediated by markers of problem-solving success.

Some support

Some support

Yes

Some support

Some support 
Figure 1. Summary of hypotheses for Study 1.

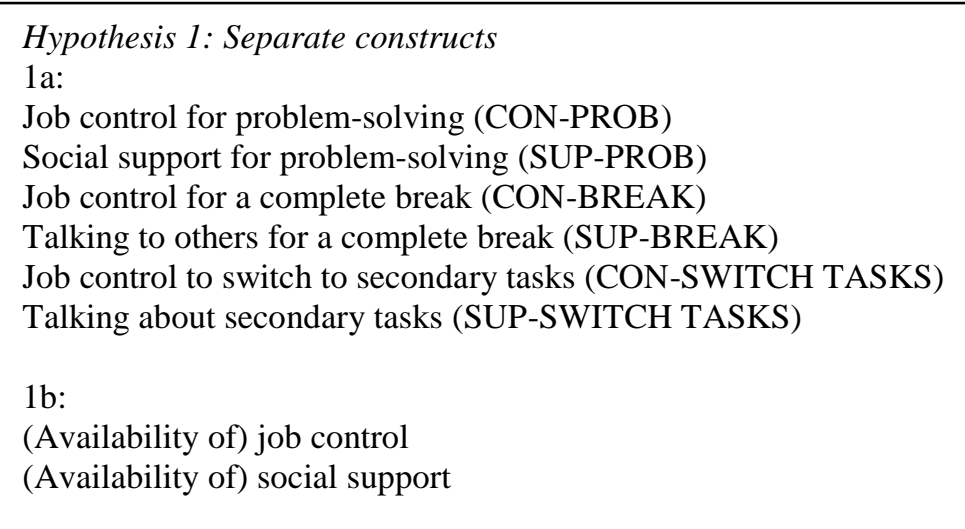

\section{Hypothesis 2:}

2a:

Job control $\leftrightarrow$ CON-PROB $>$ Job control $\leftrightarrow$ SUP-PROB

Job control $\leftrightarrow$ CON-BREAK $>$ Job control $\leftrightarrow$ SUP-BREAK

Job control $\leftrightarrow$ CON-SWITCH TASKS $>$ Job control $\leftrightarrow$ SUP-SWITCH TASKS

2b:

Social support $\leftrightarrow$ SUP-PROB $>$ Job control $\leftrightarrow$ SUP-PROB

Social support $\leftrightarrow$ SUP-BREAK $>$ Job control $\leftrightarrow$ SUP-BREAK

Social support $\leftrightarrow$ SUP-SWITCH TASKS $>$ Job control $\leftrightarrow$ SUP-SWITCH TASKS

\section{Hypothesis 3:}

3a: CON-PROB $\leftrightarrow$ SUP-PROB > CON-PROB $\leftrightarrow$ SUP-BREAK or SUP-SWITCH TASKS

3b: CON-BREAK $\leftrightarrow$ SUP-BREAK $>$ CON-BREAK $\leftrightarrow$ SUP-PROB or SUP-SWITCH TASKS

3c: CON-SWITCH TASKS $\leftrightarrow$ SUP-SWITCH TASKS > CON-SWITCH TASKS $\leftrightarrow$ SUP-PROB or SUP-BREAK

Hypothesis 4:

4a: CON-PROB and SUP-PROB $\leftrightarrow$ problem-solving demands

4b: CON-PROB, SUP-PROB, CON-SWITCH TASKS and SUP-SWITCH TASKS $\leftrightarrow$ problem-solving demands

4c: CON-BREAK and SUP-BREAK $\leftrightarrow$ fatigue, negative affect, positive affect

4d: CON-SWITCH TASKS and SUP-SWITCH TASKS $\leftrightarrow$ fatigue, negative affect, positive affect

4e: Job control $\leftrightarrow$ criterion variables $\neq$ CON-PROB, CON-BREAK or CON-SWITCH TASKS $\leftrightarrow$ criterion variables

4f: Social support $\leftrightarrow$ criterion variables $\neq$ SUP-PROB, SUP-BREAK or SUP-SWITCH TASKS $\leftrightarrow$ criterion variables

Research question 5:

CON-PROB, CON-BREAK and CON-SWITCH TASKS $\leftrightarrow$ criterion variables

$\neq$

SUP-PROB, SUP-BREAK or SUP-SWITCH TASKS $\leftrightarrow$ criterion variables 